\title{
The game of arboricity
}

\author{
Tomasz Bartnicki ${ }^{1}$, Jarosław Grytczuk ${ }^{1}$ and Hal Kierstead ${ }^{2}$ \\ ${ }^{1}$ Faculty of Mathematics, Computer Science and Econometrics, University of Zielona Góra, 65-516 Zielona Góra, \\ Poland,t.bartnicki@wmie.uz.zgora.pl,j.grytczuk@wmie.uz.zgora.pl \\ ${ }^{2}$ Department of Mathematics and Statistics, Arizona State University, Tempe, Arizona 85287, U.S.A., Kierstead@asu.edu
}

Using a fixed set of colors $C$, Ann and Ben color the edges of a graph $G$ so that no monochromatic cycle may appear. Ann wins if all edges of $G$ have been colored, while Ben wins if completing a coloring is not possible. The minimum size of $C$ for which Ann has a winning strategy is called the game arboricity of $G$, denoted by $A_{g}(G)$. We prove that $A_{g}(G) \leq 3 k$ for any graph $G$ of arboricity $k$, and that there are graphs such that $A_{g}(G) \geq 2 k-2$. The upper bound is achieved by a suitable version of the activation strategy, used earlier for the vertex coloring game. We also provide other strategie based on induction.

\section{Introduction}

We consider the following graph coloring game. Ann and Ben alternately color the edges of a graph $G$ using a fixed set of colors $C$. The only restriction they both have to respect is that no monochromatic cycle may be created. Ann wants to accomplish a coloring of the whole graph $G$, while Ben aims to achieve a partial coloring that would not be extendable without violating the acyclicity condition. The minimum size of $C$ guaranteeing a win for Ann is the game arboricity of $G$, which we denote by $A_{g}(G)$. Clearly, $A_{g}(G)$ is at least $A(G)$ - the usual arboricity of a graph $G$, that is, the minimum number of forests needed to cover the edges of $G$.

\section{Results}

Let $L(G)$ denote the minimum of the largest vertex outdegree taken over all orientations of a graph $G$. Our main result [2] is

Theorem 1 For any graph $G, A_{g}(G) \leq 3 L(G)$ holds.

Proof: (Sketch) The key idea is to develop a suitable edge version of the "activation strategy", used earlier for different variants of the vertex coloring game (see [6], [9]). To do this we introduce auxiliary edgemarking game on a directed graph $\vec{G}=(V, \vec{E})$. The main difference in our aproach is that no prespecified order of the edge set is needed.

Corollary 1 If $G$ is a planar graph then $A_{g}(G) \leq 9$.

Theorem 2 For each positive integer $k$, there exists graph $G$ with $A(G) \leq k$ and $A_{g}(G) \geq 2 k-2$. 
Proof: (Sketch) We construct suitable graph $G$ with $A(G)=k$ and then we describe strategy for Ben. Let $n=2 k$ be an even positive integer. Let $H=(V \cup I, E)$ be a graph such that $G[V]=K_{n}, G[I]$ is edgeless, and for each $k$-subset $S$ of $V$ there are $k^{2}$ vertices in $I$ whose neighborhood is $S$. We call this graph the $k$-hedgehog. The arboricity of the $k$-hedgehog is equal to $k$, but Ben has a strategy which forces at least $2 k-2$ colors.

Theorem 3 If $G$ is a 2-degenerate graph then $A_{g}(G) \leq 2$.

Proof: (Sketch) We shall prove it by induction on the number of edges. The induction step is based on the observation that each 2-degenerate graph $G$ contains a non-separable pair of edges (such that each cycle through one of them contains the other one).

Corollary 2 If $G$ is an outerplanar graph then $A_{g}(G) \leq 2$.

\section{Remarks}

Our results show that $A_{g}(G)$ behaves more tamely than $\chi_{g}(G)$. In fact, the difference between chromatic number $\chi(G)$ and game chromatic number $\chi_{g}(G)$ can be arbitrarily large already for bipartite graphs. Also some questions which are hard for the vertex game can be easily answered (at least for some classes of graphs) using our results. For instance, in [9] Zhu asked whether the fact that Ann wins the vertex game with $k$ colors on a graph $G$ implies that she wins with $k+1$ colors, too. At first glance the question looks like a joke-the more colors, the better for Ann. However, despite some efforts, no proof was supplied so far. For arboricity game Zhu's question has a positive answer for 2-degenerate graphs. Also a weaker version of the problem, which asks for a function $f(k)>k$ such that Ann wins with $f(k)$ colors provided she wins with $k$ colors, is easily answered by our theorem, as we may take $f(k)=3 k$. A nice challenging problem would be to determine the game arboricity of planar graphs. The above result implies that $A_{g}(G) \leq 9$ for any planar graph $G$ which does not seem to be the best possible bound.

Let $g(k)$ be the maximum of $A_{g}(G)$ over all graphs of arboricity at most $k$. By our results we have $2 k-2 \leq g(k) \leq 3 k$. It would be interesting to find out which of these two bounds is closer to the truth. The result of Theorem 3 suggest that perhaps the following conjecture holds.

Conjecture $1 A_{g}(G) \leq d$, for any d-degenerate graph $G$.

\section{References}

[1] T. Bartnicki, J. Grytczuk, A note on the game chromatic index of graphs, submitted.

[2] The game of arboricity, submitted.

[3] H. L. Bodlaender, On the complexity of some coloring games, Internat. J. Found. Comput. Sci. 2 (1991), 133-147.

[4] T. Dinski, X. Zhu, A bound for the game chromatic number of graphs. Discrete Math. 196 (1999), no. 1-3, 109-115.

[5] M. Gardner, Mathematical games, Scientific American (1981). 
[6] H. A. Kierstead, A simple competitive graph coloring algorithm, J. Combinatorial Theory (B) 78 (2000), 57-68.

[7] H. A. Kierstead, W. T. Trotter, Planar graph coloring with an uncooperative partner, J. Graph Theory 18, No. 6 (1994), 569-584.

[8] H. A. Kierstead, D. Yang, Orderings of graphs and game coloring numbers, Order 20 (2003), 255264.

[9] X. Zhu, The game coloring number of planar graphs, J. Combinatorial Theory (B) 75 (1999), 245258.

[10] X. Zhu, Refined activation strategy for the marking game, preprint, 2003. 
